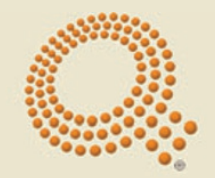

QUANTUMDロT

\title{
Western blot analysis with quantum dot fluorescence technology: a sensitive and quantitative method for multiplexed proteomics
}

\begin{abstract}
Richard L Ornberg, Theresa F Harper \& Hongjian Liu
Western or immunoblotting analysis of protein expression in cells and tissues has been the major analytical tool for assessing molecular biological functions in basic cell biological research, pathology and drug discovery and development. With the rapid growth of our understanding of gene and protein expression pathways and limited supplies of tissue, there is an ever-increasing need for more sensitive, quantitative methods capable of multiplex detection of proteins or protein states from a single western blot. Quantum Dot Corporation's (QDC) new western blot detection kits harness the high brightness, extreme stability and multiple colors of the Qdot Secondary Antibody Conjugates to quantitatively detect several proteins in a single gel. When used in combination with existing user-defined primary antibodies, the kit combines ease of use with sensitivity to picogram levels of protein using several existing gel imaging systems.
\end{abstract}

\section{Qdot western detection technology}

QDC has recently introduced two western blotting kits. These kits use Qdot Secondary Antibody Conjugates to provide a sensitive method of detecting immunolabeled proteins in western blotting applications. Qdot Conjugates are engineered fluorescent materials consisting of a Quantum dot nanocrystal core and shell of CdSe and ZnS, respectively. The nanocrystal is coated with an organic molecular layer that provides water solubility and conjugation sites for biomolecules. The fluorescence properties of the quantum dot core are one of the key advantages for its use in western blot applications over currently used detection technology. Quantum dots absorb light over a broad spectral range and fluoresce at wavelengths determined by their physical size ${ }^{1,2}$. QDC has developed processes to produce precise size distributions and consequently precise, narrow spectral emissions ranging from 525 to $800 \mathrm{~nm}$. The narrow emission enables simplified multiplexed labeling, image acquisition and quantification. Qdot nanocrystals are extremely efficient at absorbing light and converting it to a highly stable fluorescent emission, making them up to $50 \times$ brighter than conventional organic fluorophores. This high level of brightness and excellent photostability bring the added value of high sensitivity and ruggedness.

Qdot Western Blotting Kits are designed to be a 'plug-and-play' substitute for colorimetric or chemiluminescent detection systems on blots labeled with rabbit and mouse primary antibodies. Electroblotting from SDS-PAGE gels is performed using routine

Quantum Dot Corporation, 26118 Research Road, Hayward, California 94545 USA.

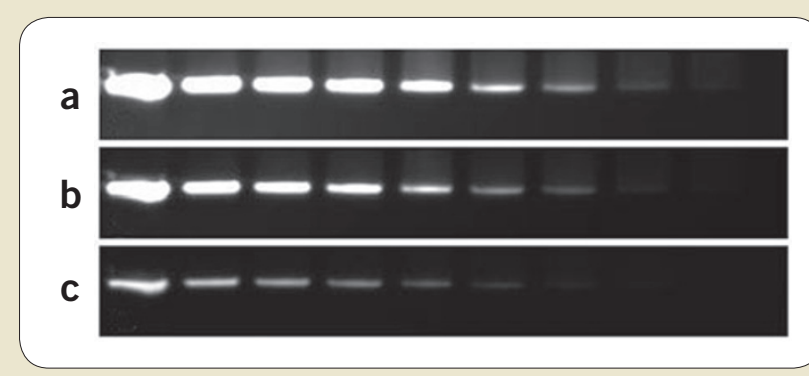

Figure 1 | Western blot of p42 MAPK recombinant protein. I mages acquired with different time exposures of a blot containing twofold serial dilutions of recombinant p42 MAPK protein and labeled with a rabbit anti-pan Erk primary antibody followed by Qdot $705 \mathrm{~nm}$ anti-Rabbit Secondary Conjugate. The dilution range is from $5 \mathrm{ng}$ (lane 1) to $20 \mathrm{pg}$ (lane 9) per lane. I mages were acquired for $5 \mathrm{~min}(\mathbf{a}), 2 \mathrm{~min}(\mathbf{b})$ and $0.6 \mathrm{~min}$ (c). Measures of the mean band intensities are shown in Figure 2.

procedures, and the blots are labeled with rabbit or mouse primary antibodies at concentrations typically used for colorimetric or chemiluminescent detection. The blots are subsequently labeled with Qdot Conjugates and imaged with one of several fluorescence gel imaging systems. The procedure requires no additional time other than what is typically done for simple colorimetric detection. Labeled blots can be stored in a buffer at $4-8{ }^{\circ} \mathrm{C}$ with minimal loss of signal for imaging at a later date.

Two western blot kits are available, one containing Qdot 565 $\mathrm{nm}$ Goat anti-Mouse Conjugate and Qdot $655 \mathrm{~nm}$ Goat antiRabbit Conjugate and a second that contains Qdot $605 \mathrm{~nm}$ Goat 

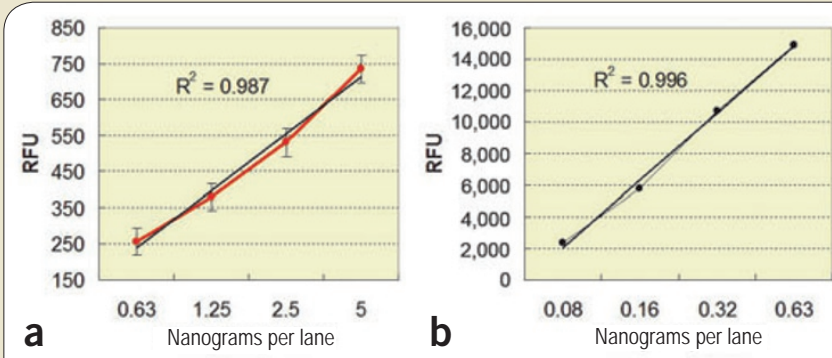

Figure 2 | Quantitative analysis of western blot of p42 MAPK protein. Graph of mean band intensity of recombinant p42 MAPK from western blot images acquired at (a) $0.6 \mathrm{~min}$ and (b) $2 \mathrm{~min}$ exposure shown in Figure 1. Blots were immunolabeled with rabbit anti-p42 MAPK primary antibody followed by Qdot $705 \mathrm{~nm}$ anti-Rabbit Secondary Conjugate. I mages were acquired on a Kodak MM2000 gel documentation station and analyzed for mean intensity above background.

anti-Mouse Conjugate and Qdot $705 \mathrm{~nm}$ Goat anti-Rabbit Conjugate. Although additional colors are available, two colors have been provided to enable flexible multiplex labeling and quantification with existing gel imaging systems. Each kit is supplied with all the necessary reagents to label ten blots. The kit includes Qdot Conjugates, block and wash buffers and PVDF blotting membranes specially selected for low auto-fluorescence. All reagents have been optimized for high sensitivity and low background signal with mouse and rabbit primary antibodies.

\section{Qdot western blot imaging}

Qdot western blot imaging is flexible and can be done at several levels of sophistication. At a minimum, a hand-held blue or ultraviolet light source and a high-quality color digital camera can be used to illuminate the blot and capture images of the fluorescent protein bands. With a gel imaging or documentation workstation, detection varies with the illumination source and the installed filters on that particular model of the instrument. Most gel imagers have a source sufficient to excite Qdot Conjugates, such as an epi-UV illuminator. This source provides adequate excitation and minimal auto-fluorescence over transilluminating UV sources. However, it is recommended that an epi-illuminator source equipped with a $100 \mathrm{~nm}$-wide bandpass filter centered at about $415 \mathrm{~nm}$ be used, particularly for the $705 \mathrm{~nm}$ emission, as this source has no unfiltered near-infrared emission that obscures $705 \mathrm{~nm}$ detection. For optimized sensitivity and multiplexed detection, emission filters specific for Qdot nanocrystals are recommended. These filters can be obtained from Omega Optical and have to be ordered in sizes appropriate to the particular instrument.

\section{Performance and sensitivity}

The Qdot Western Blotting Kits have been rigorously designed to maximize sensitivity and minimize both nonspecific binding of the conjugate and blotting membrane auto-fluorescence. Fluorescence detection with Qdot Western Blotting Kits has several advantages over colorimetric and chemiluminescent techniques in that the range of linear sensitivity of detection can be adjusted with the image exposure time, a distinct benefit of Qdot Conjugate photostability. Short integration times can be used to capture measurable data from more abundant proteins, and longer times can be used to extend the range of linear data to less abundant proteins. For example, a blot with serial dilutions of the 442 mitogen-activated phosphokinase (p42 MAPK) protein from $5 \mathrm{ng}$ to $20 \mathrm{pg}$ per lane was imaged for 5, 2 and 0.6 min (Fig. 1). At 2 and 5 min exposure times, lanes containing protein in excess of $600 \mathrm{pg}$ were saturated, but lanes containing lower concentrations of protein were not saturated and suitable for quantitative measurements. Shorter exposure times (0.6 $\mathrm{min}$ ) produce unsaturated images of bands with high amounts of protein. By selecting appropriate exposure times, Qdot Western Blotting Kits can provide accurate quantitative data over a broad range of protein loading concentrations. Standard gel image analysis routines can be used to measure the mean intensity in a band. The 0.6 min exposure of the p42 MAPK blot (Fig. 1c) produced a linear response for protein concentrations ranging from 5 to 0.6 ng per lane (Fig. 2a). After longer exposure ( $2 \mathrm{~min}$ ) the linear concentration range was shifted down to the 600-60 pg range (Fig. 2b). More sophisticated image acquisition and analysis methods are likely to improve these results.

The minimum amount of protein that can be detected with the Qdot Western Blotting Kit is a function of antibody affinities and the sources of background fluorescence in the image, such as the nonspecific binding of the primary antibody and the auto-fluorescence of the blotting membrane. Several protein-antibody combinations have shown sensitivities to the low picogram level with a 100 -fold linear concentration range.

\section{Applications}

The Qdot Western Blotting Kit comes with two colors of quantum dots conjugated to goat $\mathrm{F}\left(\mathrm{ab}^{\prime}\right)_{2}$ anti-mouse and goat $\mathrm{F}\left(\mathrm{ab}^{\prime}\right)_{2}$ antirabbit IgG. This configuration provides the capability of doing

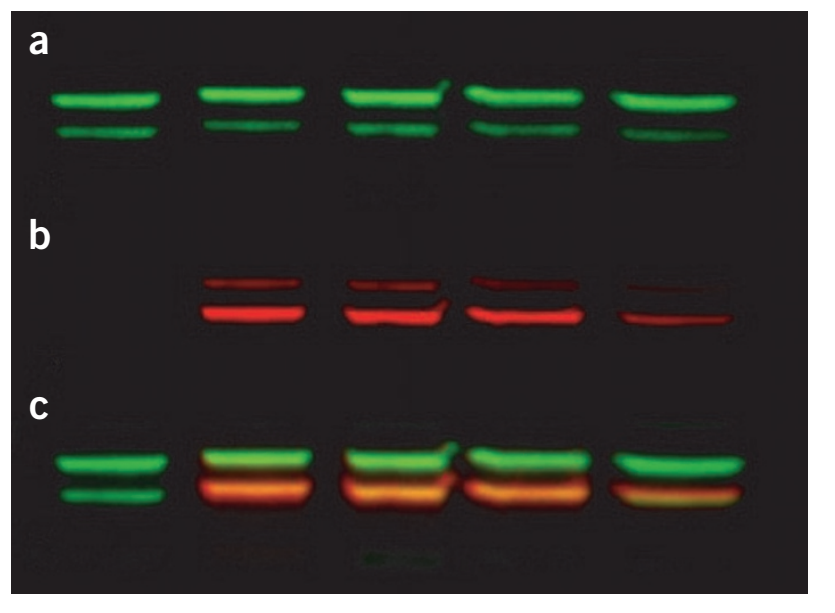

Figure 3 | Multiplex western blot describing kinetics of p42 MAPK phosphorylation in PDGF-treated NIH 3T3 cells. Western blot images of p42 MAPK (a, green) and phosphorylated p42 MAPK ( $\mathbf{b}$, red) expression in serumstarved 3T3 cells in response to PDGF stimulation. Cells were harvested at 0 , 10, 20, 40 and 60 min after PDGF administration (lanes 1-5, respectively). The anti-pan MAPK primary antibody (a) was detected with Qdot $605 \mathrm{~nm}$ Conjugate, a red-orange dot that was pseudocolored green and the phosphoMAPK primary antibody (b) was detected with Qdot $705 \mathrm{~nm}$ Conjugate and pseudocolored red. (c) Overlay of images in $\mathbf{a}$ and $\mathbf{b}$. 
ADVERTISING FEATURE

APPLI CATION NOTES

sensitive multiplex detection of several proteins on a single blot. Using a pair of primary antibodies to different epitopes on a single protein where one of the pair is a mouse antibody and the other is a rabbit antibody, a variety of proteomics analyses are possible, including analyses of differential expression and post-translational modifications such as glycosylation and phosphorylation or dephosphorylation activity. For example, the kinetics of p42/44 MAPK phosphorylation following platelet-derived growth factor (PDGF) stimulation in serum-starved 3T3 cells can be observed in a sin-

을 gle blot using secondary antibody conjugates to a mouse anti-pan MAPK antibody and a rabbit anti-phospho MAPK antibody (Fig. 3). Hence, multiplex labeling with secondary antibody conjugates provides all of the information that would typically require two blots with colorimetric or chemiluminescent detection. Rabbit and mouse antibody pairs to a large number of signal transduction proteins are available from antibody suppliers for enzyme linked immunosorbent assay (ELISA) applications and western labeling. For primary antibodies other than rabbit or mouse, other Qdot Conjugates to goat human and chicken antibodies can be substituted in the kits. With the Qdot Western Blotting Kit, analysis of signal transduction and other protein modification activities can be greatly accelerated with high sensitivity and accurate quantification.

Conclusion

The Quot Western Blotting Kit brings the power of quantum dot fluorescence technology to a work-horse application in proteomics. The advantages of high sensitivity, multiplex labeling, photo- and chemical stability and the availability of many compatible gel maging platforms will accelerate information flow in proteomics.

1. Alivisatos, A.P. Semiconductor clusters, nanocrystals, and quantum dots. Science 271, 933-937 (1996).

2. Bruchez, M.P., Moronne, M., Gin, P., Weiss, S. \& Alivisatos, A.P. Semiconductor nanocrystals as fluorescent biological labels. Science 281, 2013-2016 (1998).

NATURE METHODS | VOL NO | JANUARY 2005 | 81 